\title{
DESIGNING CROSSWORD PUZZLE TO IMPROVE STUDENTS' VOCABULARY MASTERY OF THE THIRD SEMESTER IN ENGLISH EDUCATION DEPARTMENT STUDENT OF UIN ALAUDDINMAKASSAR
}

\author{
Sukirman \\ Asti Astuti Ningsih \\ English Education Department of UIN Alauddin Makassar \\ sukirmanmarshan.sm@gmail.com \\ astiastutiningsib@gmail.com
}

\begin{abstract}
This research design Crossword Puzzle in the Third Semester of English Education Department at UIN Alauddin Makassar. The objective of this research was to design crossword puzzle to improve students' vocabulary mastery. It was conducted in the third semester students in the English Education Department of UIN Alauddin. The significance of this research were for students, teachers, and other research. For students with their research will facilitate the student in improving their vocabulary. In addition, this study was designed to produce teaching materials, especially in vocabulary lesson used crossword puzzle. For teachers, this research can be useful to produce media that can facilitate teachers in presenting the lesson. For other researchers, the results of this study can be used as a comparison, especially in terms of the development of teaching materials in vocabulary. The research design of this research was Research \& Development (R\&D). It applied model R\&D adapted from Borg \& Gall model. The steps of the model were needed assessment, designing the product, validating product, revising initial product, trying out product, final revising product, and the last final product. The types of data of this research were quantitative and qualitative data. The research instruments were questionnaire and research judgments.
\end{abstract}

KEYWORDS: Designing, Crossword Puzzle, Vocabulary Mastery

\section{A. INTRODUCTION}

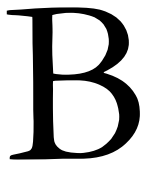

ased on an interview that the researcher conducted in January 2015 at English Education Department students, they said that generally when they learnt vocabulary in the classroom, they accept the lesson maximally $70 \%$. However, sometimes they do not understand how to use the words in a sentence and also do not understand in classifying words. Another problem is they difficult to produce the words because lack of attention about how to pronoun words from the lecturer. Based on the problem above, researchers conclude that the students still need to develop their ability to master vocabulary and we need an appropriate method based on students' need to make the learning process more effective and reach a learning objective.

There were many factors affecting the students were not interested in studying vocabulary. First, the ways to produce the words were different from the text. Second, the 
methods that the teacher applied in the classroom for learning vocabulary were monotonous. Besides that, vocabulary was the basic way to learn English. Third, students were also difficult to pronounce the words in English because they were not accustomed in English language caused their environment did not use English as daily speaking.

In this case, when students were interested in learning English by developing their vocabulary, they will get the basic knowledge in learning English. Vocabulary was the primary importance to language teaching and learning because it plays a pivotal role in mastery four language skills: reading, writing, speaking and listening. When students know vocabulary, they got easier to study another part of the English language.

From the problem above, the researcher designed a new media to improve students' mastery in vocabulary namely crossword. It was a kind of games that researcher designed and developed for students in English Education Department as a learning media. This media were given to student in second semester.

\section{B. LITERATURE RIVIEW}

\section{Designing}

Designing is an idea to developing a new crossword puzzle media appropriate with students' need used kinds model of puzzle to interest students in learning vocabulary. Designing also need creativity and well knowledge to make the design up to date. Big Indonesian Dictionary (2008: 346), the design is the initial idea, design, planning arrangement pattern, form the framework of a building, the building motif, pattern building. Design is a creative activity that brings updates (Reswick, 1965). The design can also be interpreted as a creative artist to meet the specific needs and certain ways. Designs can also be a problem solving with a clear target (Archer, 1965). Meanwhile, according to Alexander (1963) finding the physical element of the design is the most objective.

\section{Crossword Puz:le}

Crossword puzzle is letter games and a form of mind sport (Uberman: 20014). Arthur Wyne published crossword puzzle in New York world, which embodied most of the features of the genre. This puzzle is frequently cited as the first crossword puzzle and Wyne as the inventor.

Crossword puzzle takes a form as square grid of black and white squares. They play is to fill the white square with letters, forming words reading across and down, by solving the clues which yield the words. Boxes in the crossword puzzle are given number left to right and up to bottom. The numbers then refers for the clues. In almost cases, the grid is rationally symmetric.

Plaister in Reny (2005: 18) said that there are some advantages that can be taken from use crossword puzzle in teaching vocabulary. The advantages are, a)it can increase students' vocabulary acquisition because this technique the students are learning by doing. 
As we know that learning by doing is more crossword puzzle by them, students try meaningful in motivating by completing the tofind the new vocabulary and keep remember them; b) by solving the crossword puzzle, the students trained to involving in problem solving. For the teacher, these techniques can also solving the problem of monotones and boring class; c)it is interesting and challenging for the students. It can be avoid students' boredom from a monotones technique. In this case, crossword puzzle tries to increase students' attention to the lesson materials and this produce better learning; d) by solving the crossword puzzle in group or classroom discussion students' are trained to more critical in thinking; e) working the crossword puzzle in group in the re lax atmosphere will make the students feel fun and enjoy the learning. This is meaningful and helpful inlearning a language and it can encourage all of the students to participate in learning process.

\section{Vocabulary}

There is no unanimity as to the precise definition of the term vocabulary. For example, Graves (2000) defines vocabulary as the entire stock of words belonging to a branch of knowledge or known by an individual. Harmer (1993) claims that at the basic level, knowing a word involves knowing its form and its meaning while at a deeper level, it means the abilities to know its meaning, usage, word formation, and grammar. Vocabulary is not only confined to the meaning of words, but it also includes how vocabulary in a language is structured, how people use ad store words and how they learn words and the relationship between words, phrases, categories of words and phrases (Harmer:1993). Nation (2001), on the other hand, argues that acquiring a word means knowing its form (spoken, written and word parts); meaning (concept and associations); and use in terms of grammatical functions, collocations and constraints.

According to Hornby (1983) state that vocabulary is a total number of words which make up a language. It also defines as a body of words known to a person or used in a particular book, subject.List of words with their meanings, especially one which accompanies a text book in a foreign language.

Best on some definition previous the writer concludes that vocabulary is not merely a list of words with definition or a stock of words know to a person but also every word that we find, listen, or a stock of words know to a person but also every word that we find, listen, or that used in speaking, reading and writing.

\section{RESEARCH METHOD}

The researcher conducted a Research and Development (R\&D). Research and Development was a research method used to result a certain product and examine the appropriateness or effectiveness of the product.

Types of data are being obtained on this research is qualitative andquantitative. 
Qualitative data is taken from expert judgment. Every result of design crossword puzzle is given to expert and they will examine it until the design valid and appropriate for use in class. Quantitative data is taken from analyzing students' score after trying out the crossword puzzle design.

The researcher utilized two kinds of instrumental to collect the data from the subject. The researcher will use questionnaire and expert judgment in this research.

The questionnaire was given to the students in thethird semester of English Education Department UIN Alauddin Makassar. The form of questionnaire was check list questionnaire where respondent only check their answer in to appropriate column. This questionnaire were consists of ten questions. All questions are given to get some data about how far student's mastery vocabulary in learning process before try out crossword puzzle design.

An expert judgment was given to an expert person who have much knowledge in designs' validity. Expert judgments will evaluate the design of crossword puzzle based from the rubric. The rubric are, 1) the use of letter; 2) the typing; 3) the use of an appropriate spacing; 4) the use of layout; 5) the use of creative layout; 6) the use of an appropriate picture; 7) the design of puzzle; 8) the appropriate content; 9) the use of understable language; and 10) the use of understable instruction.

To collect data needed by the researcher, the researcher carrie out the following procedures based on the Research and Development:

1. Observing and analysing needs fordesigning crossword puzzle of students' vocabulary mastery.

2. Making initial report (draft) and research planning

3. Designing Crossword Puzzle for improving students' vocabulary mastery.

4. Validating design of the Crossword Puzzle by experts

5. Revising the Crossword Puzzle based on the experts.

6. Trying out the Crossword Puzzle to third semester students of English Education Department in UIN 
7. Revising the Crossword Puzzle based on the result of try out become final product

8. Analysing and writing the result of designing Crossword Puzzle.

Figure 1. Procedure of Collecting Data based on R\&D

For analyzing the data, the researcher used validity and realibility.

\section{Validation product design by experts.}

The data analysis of questionnaire about the experts of the Crossword puzzle was done by percentage description technique. The score which was get from all aspect will be counted by formula. (Purwanto, 2004 in Khikmah 2013)

$$
\begin{aligned}
N P & =\frac{R}{S M} \times 100 \% \\
\mathrm{NP} & =\text { the searched percent value } \\
\mathrm{R} & =\text { obtained score } \\
\mathrm{SM} & =\text { maximum score }
\end{aligned}
$$

Table 1. Expediency level criteria of the product

\begin{tabular}{|c|c|}
\hline Criteria interval & Criteria \\
\hline $81 \% \leq \mathrm{NP} \leq 100 \%$ & Very proper \\
$62 \% \leq \mathrm{NP} \leq 81 \%$ & Proper \\
$43 \% \leq \mathrm{NP} \leq 63 \%$ & Quite proper \\
$33 \% \leq \mathrm{NP} \leq 43 \%$ & Less proper \\
$\mathrm{NP} \leq 33 \%$ & Not proper \\
\hline
\end{tabular}

\section{Validity of Crossword Puzzle}

Validate each question of the product carried out by used correlation formula of product moment. (Arikunto, 2013 in Noveria 2015).

$$
r_{x y}=\frac{N \sum X Y-\left(\sum X\right)\left(\sum Y\right)}{\sqrt{\left[( N \sum X ^ { 2 } - ( \sum X ^ { 2 } ) ) \left[\left(N \sum Y^{2}-\left(\sum Y^{2}\right)\right]\right.\right.}}
$$

In which:

$$
\begin{aligned}
& \mathrm{rxy}=\text { Correlation coefficient } \\
& \mathrm{N}=\text { The number of the testee }
\end{aligned}
$$


Sukirman \& Asti Astuti Ningsih,Designing Crossword Puzzle to Improve Students' Vocabulary Mastery ...

$$
\begin{aligned}
& \mathrm{X}=\text { Score of Variable } 1 \\
& \mathrm{Y}=\text { Score of Variable } 2
\end{aligned}
$$

The validity could be found out by the classification of validity index as follows:

Table 2: Validity Index

\begin{tabular}{|c|c|}
\hline The Amount of Validity & Interpretation \\
\hline $0,800-1,00$ & Excellent \\
\hline $0,600-0,800$ & Good \\
\hline $0,400-0,600$ & Satisfactory \\
\hline $0,200-0,400$ & Poor \\
\hline $0,00-0,200$ & Very Poor \\
\hline
\end{tabular}

(Arikunto, 2003 in Noveria 2015)

Besides the index above, Arikunto (2003) in Noveria (2015) states that if the result of $r$ in a test item was higher than table of Product Moment, it means that the item was considered to be valid. This way was more up-to date than using such index above.

\section{Reliability of Crossword Puzzle}

The reliability of each item was analyzed by using coefficient formula Alpha Cronbach. (Arikunto, 2013: 223)

$$
\text { In which: } r_{11=\frac{2 \times r 1 / n 1 / n}{1+r 1 / n 1 / 2}}
$$

$r_{11}=$ Reliability

$r 1 / 21 / 2=$ rxy mentioned as correlation index

Arikunto (2006:184) also states that if the result of $r_{11}$ in a test item is higher than table of product moment, it means that item is considered to be reliable.

\section{FINDINGS AND DISCUSSION}

\section{Need Assessment}

Before designing the crossword puzzle to improving students' vocabulary mastery, the researcher had done observation of third semester's students of the English Education Department at UIN Alauddin Makassar. The researcher had observed the students about what method that lecturer usually used in teaching vocabulary and how far the students can accept vocabulary materials during the learning process by do some interview. The researcher also observed about how far students mastery in vocabulary by using a questionnaire. This questionnaire was given to 40 students in the third semester of English 
Education Department at UIN Alauddin Makassar. Based on the data which the researcher had gained from the third semester students, the researcher had found that there were still many students do not understand how to use the words in a sentence and also do not understand in classifying words. And after observing the students' answer in the questionnaire, there were also still many students did not know about academic writing vocabulary. Besides academic writing, students also difficult to memorize verb and adjective vocabulary. In addition, $80 \%$ student prefers using games' method in learning vocabulary.

\section{Designing Product}

The researcher had designed a crossword puzzle to improve students' vocabulary mastery. Because the crossword puzzle dealing to improve students' mastery, so it was designed based on students' need. The researcher has made an observation about what students' need through a questionnaire. After seeing the students' questionnaire, it showed that $80 \%$ students prefer to learn vocabulary by using game. The crossword puzzle was kinds of game that help students easy in learning vocabulary. This crossword puzzle also measures how far students can improve their vocabulary mastery. The crossword puzzle also deals with the blueprint which had been made by the researcher. The blueprint consists of kinds vocabulary that students have to answer. Kinds of vocabulary that researchers used in design crossword puzzle are adjective, verb (irregular and regular), noun (abstract, food, place, people, animal, nature and etc and also academic writing. The result of this step produces the crossword puzzle for improving students' vocabulary mastery in two models of puzzle which each model consists of six kinds of puzzle. In the first model, each puzzle consist of ten questions. Then for the second model, each puzzle consist of fifteen questions.

\section{Validating Product}

The initial design of the crossword puzzle was validated by an expert. The expert was a lecturer in the English Education Department at UIN Alauddin Makassar. The result of the expert validating was provided an expert was calculated based on the formula according to Purwanto (2004) in Sri (2015). The result of validating product can be calculated as follows:

1) The validation result of expert.

$$
N P=\frac{83}{84} \times 100 \%=98 \%
$$

In which:

$\mathrm{NP}=$ the searched percent value

$83=$ obtained a score $(\mathrm{R})$

$84=$ maximum score $(\mathrm{SM})$ 
Based on the calculation above, the value of the validation which obtained was $98 \%$. In the table of expediency level criteria of the product by value, $98 \%$ means that the product is very proper

\section{Revision Initial Product}

\begin{tabular}{|l|l|l|}
\hline No. & Suggestion & Revision \\
\hline 1. & $\begin{array}{l}\text { The direction in crossword puzzle still } \\
\text { unclear and make students difficult to } \\
\text { answer the puzzle. }\end{array}$ & $\begin{array}{l}\text { Make one chapter to introduce students } \\
\text { about crossword puzzle and how to } \\
\text { answer crossword puzzle. }\end{array}$ \\
\hline 2. & $\begin{array}{l}\text { There were design forms which can } \\
\text { make students confused to answer } \\
\text { crossword puzzle. }\end{array}$ & $\begin{array}{l}\text { Repair the design with clear and easy for } \\
\text { students to answer the crossword puzzle. }\end{array}$ \\
\hline 3. & $\begin{array}{l}\text { There were many forms that researcher } \\
\text { design. This will make students difficult } \\
\text { to answer many questions from the } \\
\text { crossword puzzle. }\end{array}$ & $\begin{array}{l}\text { The researcher reduces design of } \\
\text { crossword puzzle from 24 design form } \\
\text { which consist of 300 number of questions } \\
\text { become 12 design forms which consist of } \\
150 \text { number of questions. }\end{array}$ \\
\hline
\end{tabular}

\section{Trying Out the product}

The product tried out in the third semester students' in English Education Department of UIN Alauddin Makassar.

1) Validity

The Result ofV alidity of crossword puzzle first model by Table of Product Moment

1. Crossword Adjective

\begin{tabular}{|c|c|c|c|c|}
\hline \multirow{2}{*}{$\begin{array}{l}\text { Question } \\
\text { Number }\end{array}$} & $\begin{array}{c}\text { Correlation } \\
\text { value }\end{array}$ & $\begin{array}{c}\text { Comparative } \\
\text { Coefficient } \\
(\mathbf{r x y})\end{array}$ & $\mathbf{5 \%}$ & $\begin{array}{c}\text { Category } \\
\text { of }\end{array}$ \\
\hline 1 & 0.78 & 0.632 & rxy $>$ r value & Validity \\
\hline 2 & 0.67 & 0.632 & rxy $>$ r value & Valid \\
\hline 3 & 0.67 & 0.632 & rxy $>$ r value & Valid \\
\hline 4 & 0.73 & 0.632 & rxy $>$ r value & Valid \\
\hline 5 & 0.65 & 0.632 & rxy $>$ r value & Valid \\
\hline 6 & 0.85 & 0.632 & rxy $>$ r value & Valid \\
\hline 7 & 0.90 & 0.632 & rxy $>$ r value & Valid \\
\hline 8 & 0.64 & 0.632 & rxy $>$ rvalue & Valid \\
\hline 9 & 0.75 & 0.632 & rxy $>$ r value & Valid \\
\hline 10 & 0.71 & 0.632 & rxy $>$ r value & Valid \\
\hline
\end{tabular}

2. Crossword Verb

\begin{tabular}{|l|l|c|c|c|}
\hline $\begin{array}{l}\text { Question } \\
\text { Number }\end{array}$ & $\begin{array}{c}\text { Correlation } \\
\text { Coefficient }\end{array}$ & $\begin{array}{c}\mathbf{r} \\
\text { value } \\
\mathbf{5} \%\end{array}$ & $\begin{array}{c}\text { Comparative } \\
\text { between rxy and } \mathbf{r}\end{array}$ & $\begin{array}{c}\text { Category } \\
\text { of } \\
\text { Validity }\end{array}$ \\
\hline
\end{tabular}




\begin{tabular}{|c|c|c|c|c|}
\hline & $(\mathbf{r x y})$ & & value & \\
\hline 1 & 0.64 & 0.632 & $\operatorname{rxy}>$ r value & Valid \\
\hline 2 & 0.83 & 0.632 & rxy $>$ rvalue & Valid \\
\hline 3 & 0.67 & 0.632 & rxy $>$ r value & Valid \\
\hline 4 & 0.70 & 0.632 & rxy $>$ rvalue & Valid \\
\hline 5 & 0.84 & 0.632 & rxy $>$ r value & Valid \\
\hline 6 & 0.83 & 0.632 & rxy $>$ r value & Valid \\
\hline 7 & 0.89 & 0.632 & rxy $>$ rvalue & Valid \\
\hline 8 & 0.67 & 0.632 & rxy $>$ r value & Valid \\
\hline 9 & 0.83 & 0.632 & rxy $>$ r value & Valid \\
\hline 10 & 0.82 & 0.632 & rxy $>$ r value & Valid \\
\hline
\end{tabular}

3. Crossword Noun (Abstract)

\begin{tabular}{|c|c|c|c|c|}
\hline $\begin{array}{l}\text { Question } \\
\text { Number }\end{array}$ & $\begin{array}{c}\text { Correlation } \\
\text { Coefficient } \\
\text { (rxy) }\end{array}$ & $\begin{array}{c}\mathrm{r} \\
\text { value } \\
5 \%\end{array}$ & $\begin{array}{c}\text { Comparative } \\
\text { between rxy and } \mathrm{r} \\
\text { value }\end{array}$ & $\begin{array}{c}\begin{array}{c}\text { Category } \\
\text { of }\end{array} \\
\text { Validity }\end{array}$ \\
\hline 1 & 0.65 & 0.632 & rxy $>$ rvalue & Valid \\
\hline 2 & 0.87 & 0.632 & rxy $>$ r value & Valid \\
\hline 3 & 0.73 & 0.632 & rxy $>$ r value & Valid \\
\hline 4 & 0.75 & 0.632 & rxy $>$ r value & Valid \\
\hline 5 & 0.90 & 0.632 & rxy $>$ r value & Valid \\
\hline 6 & 0.85 & 0.632 & rxy $>$ r value & Valid \\
\hline 7 & 0.68 & 0.632 & rxy $>$ r value & Valid \\
\hline 8 & 0.80 & 0.632 & rxy $>$ r value & Valid \\
\hline 9 & 0.69 & 0.632 & rxy $>$ r value & Valid \\
\hline 10 & 0.73 & 0.632 & rxy $>$ r value & Valid \\
\hline
\end{tabular}

4. Crossword Noun (People)

\begin{tabular}{|c|c|c|c|c|}
\hline $\begin{array}{c}\text { Question } \\
\text { Number }\end{array}$ & $\begin{array}{c}\text { Correlation } \\
\text { Coefficient } \\
(\mathbf{r x y})\end{array}$ & $\begin{array}{c}\mathbf{r} \\
\text { value }\end{array}$ & $\begin{array}{c}\text { Comparative } \\
\mathbf{5 \%}\end{array}$ & $\begin{array}{c}\text { Category } \\
\text { of }\end{array}$ \\
\hline 1 & 0.80 & 0.632 & rxy $>$ r value & Validity \\
\hline 2 & 0.85 & 0.632 & rxy $>$ rvalue & Valid \\
\hline 3 & 0.80 & 0.632 & rxy $>$ rvalue & Valid \\
\hline 4 & 0.67 & 0.632 & rxy $>$ r value & Valid \\
\hline 5 & 0.80 & 0.632 & rxy $>$ r value & Valid \\
\hline 6 & 0.73 & 0.632 & rxy $>$ r value & Valid \\
\hline 7 & 0.71 & 0.632 & rxy $>$ r value & Valid \\
\hline 8 & 0.80 & 0.632 & rxy $>$ r value & Valid \\
\hline 9 & 0.73 & 0.632 & rxy $>$ r value & Valid \\
\hline 10 & 0.63 & 0.632 & rxy $>$ r value & Valid \\
\hline
\end{tabular}


5. Crossword Noun (Place)

\begin{tabular}{|c|c|c|c|c|}
\hline $\begin{array}{l}\text { Question } \\
\text { Number }\end{array}$ & $\begin{array}{c}\text { Correlation } \\
\text { Coefficient } \\
\quad(\mathrm{rxy})\end{array}$ & $\begin{array}{c}r \\
\text { value } \\
5 \%\end{array}$ & $\begin{array}{c}\text { Comparative } \\
\text { between rxy and } r \\
\text { value }\end{array}$ & $\begin{array}{c}\begin{array}{c}\text { Category } \\
\text { of }\end{array} \\
\text { Validity }\end{array}$ \\
\hline 1 & 0.72 & 0.632 & rxy $>$ r value & Valid \\
\hline 2 & 0.84 & 0.632 & rxy $>$ rvalue & Valid \\
\hline 3 & 0.87 & 0.632 & rxy $>$ rvalue & Valid \\
\hline 4 & 0.65 & 0.632 & rxy $>$ rvalue & Valid \\
\hline 5 & 0.72 & 0.632 & rxy $>$ r value & Valid \\
\hline 6 & 0.87 & 0.632 & rxy $>$ rvalue & Valid \\
\hline 7 & 0.71 & 0.632 & rxy $>$ r value & Valid \\
\hline 8 & 0.67 & 0.632 & rxy $>$ rvalue & Valid \\
\hline 9 & 0.69 & 0.632 & rxy $>$ rvalue & Valid \\
\hline 10 & 0.90 & 0.632 & rxy $>$ rvalue & Valid \\
\hline
\end{tabular}

6. Crossword Noun (Nature)

\begin{tabular}{|c|c|c|c|c|}
\hline $\begin{array}{c}\text { Question } \\
\text { Number }\end{array}$ & $\begin{array}{c}\text { Correlation } \\
\text { Coefficient } \\
(\mathbf{r x y})\end{array}$ & $\begin{array}{c}\mathbf{r} \\
\text { value }\end{array}$ & $\begin{array}{c}\text { Comparative } \\
\text { between rxy and } \mathbf{r} \\
\text { value }\end{array}$ & $\begin{array}{c}\text { Category } \\
\text { of }\end{array}$ \\
\hline 1 & 0.63 & 0.632 & rxy $>$ r value & Validity \\
\hline 2 & 0.64 & 0.632 & rxy $>$ r value & Valid \\
\hline 3 & 0.78 & 0.632 & rxy $>$ r value & Valid \\
\hline 4 & 0.93 & 0.632 & rxy $>$ r value & Valid \\
\hline 5 & 0.67 & 0.632 & rxy $>$ r value & Valid \\
\hline 6 & 0.71 & 0.632 & rxy $>$ r value & Valid \\
\hline 7 & 0.89 & 0.632 & rxy $>$ r value & Valid \\
\hline 8 & 0.65 & 0.632 & rxy $>$ r value & Valid \\
\hline 9 & 0.80 & 0.632 & rxy $>$ r value & Valid \\
\hline 10 & 0.90 & 0.632 & rxy $>$ r value & Valid \\
\hline
\end{tabular}

Based on the table about crossword puzzle validity above, the researcher had gathered that from the 60 number of questions, divided in six kinds of crossword puzzle. 
The result showed that all puzzle divided in 60 number of questions were valid.

The Result ofV alidity of crossword puz:le first model by Table of Product Moment

1. Crossword Verb I

\begin{tabular}{|c|c|c|c|c|}
\hline $\begin{array}{c}\text { Question } \\
\text { Number }\end{array}$ & $\begin{array}{c}\text { Correlation } \\
\text { Coefficient } \\
\text { (rxy) }\end{array}$ & $\begin{array}{c}\mathbf{r} \\
\text { value }\end{array}$ & $\begin{array}{c}\text { Comparative } \\
\text { between rxy and } \mathbf{r} \\
\text { value }\end{array}$ & $\begin{array}{c}\text { Category } \\
\text { of }\end{array}$ \\
\hline 1 & 0.75 & 0.632 & rxy $>$ r value & Valid \\
\hline 2 & 0.78 & 0.632 & rxy $>$ rvalue & Valid \\
\hline 3 & 0.67 & 0.632 & rxy $>$ r value & Valid \\
\hline 4 & 0.73 & 0.632 & rxy $>$ r value & Valid \\
\hline 5 & 0.83 & 0.632 & rxy $>$ r value & Valid \\
\hline 6 & 0.73 & 0.632 & rxy $>$ r value & Valid \\
\hline 7 & 0.64 & 0.632 & rxy $>$ r value & Valid \\
\hline 8 & 0.87 & 0.632 & rxy $>$ rvalue & Valid \\
\hline 9 & 0.67 & 0.632 & rxy $>$ r value & Valid \\
\hline 10 & 0.63 & 0.632 & rxy $>$ r value & Valid \\
\hline 11 & 0.75 & 0.632 & rxy $>$ r value & Valid \\
\hline 12 & 0.93 & 0.632 & rxy $>$ r value & Valid \\
\hline 13 & 0.64 & 0.632 & rxy $>$ r value & Valid \\
\hline 14 & 0.87 & 0.632 & rxy $>$ r value & Valid \\
\hline 15 & 0.82 & 0.632 & rxy $>$ r value & Valid \\
\hline
\end{tabular}

2. Crossword Verb II

\begin{tabular}{|c|c|c|c|c|}
\hline $\begin{array}{l}\text { Question } \\
\text { Number }\end{array}$ & $\begin{array}{c}\text { Correlation } \\
\text { Coefficient } \\
\text { (rxy) }\end{array}$ & $\begin{array}{c}\mathrm{r} \\
\text { value } \\
5 \%\end{array}$ & $\begin{array}{c}\text { Comparative } \\
\text { between rxy and } r \\
\text { value }\end{array}$ & $\begin{array}{c}\text { Category } \\
\text { of } \\
\text { Validity }\end{array}$ \\
\hline 1 & 0.75 & 0.632 & rxy $>$ rvalue & Valid \\
\hline 2 & 0.64 & 0.632 & rxy $>$ r value & Valid \\
\hline 3 & 0.69 & 0.632 & rxy $>$ r value & Valid \\
\hline 4 & 0.81 & 0.632 & rxy $>$ r value & Valid \\
\hline 5 & 0.64 & 0.632 & rxy $>$ rvalue & Valid \\
\hline 6 & 0.72 & 0.632 & rxy $>$ rvalue & Valid \\
\hline 7 & 0.65 & 0.632 & rxy $>$ r value & Valid \\
\hline 8 & 0.73 & 0.632 & rxy $>$ r value & Valid \\
\hline 9 & 0.83 & 0.632 & rxy $>$ r value & Valid \\
\hline
\end{tabular}


Sukirman \& Asti Astuti Ningsih,Designing Crossword Puzzle to Improve Students' Vocabulary Mastery ...

\begin{tabular}{|l|l|l|l|l|}
10 & 0.65 & 0.632 & rxy $>$ rvalue & Valid \\
\hline 11 & 0.68 & 0.632 & rxy $>$ rvalue & Valid \\
\hline 12 & 0.64 & 0.632 & rxy $>$ rvalue & Valid \\
\hline 13 & 0.85 & 0.632 & rxy $>$ r value & Valid \\
\hline 14 & 0.87 & 0.632 & rxy $>$ r value & Valid \\
\hline 15 & 0.94 & 0.632 & rxy $>$ rvalue & Valid \\
\hline
\end{tabular}

3. Crossword Noun

\begin{tabular}{|c|c|c|c|c|}
\hline $\begin{array}{l}\text { Question } \\
\text { Number }\end{array}$ & $\begin{array}{c}\text { Correlation } \\
\text { Coefficient } \\
\quad \text { (rxy) }\end{array}$ & $\begin{array}{c}\mathrm{r} \\
\text { value } \\
5 \%\end{array}$ & $\begin{array}{c}\text { Comparative } \\
\text { between rxy and } r \\
\text { value }\end{array}$ & $\begin{array}{c}\begin{array}{c}\text { Category } \\
\text { of }\end{array} \\
\text { Validity }\end{array}$ \\
\hline 1 & 0.65 & 0.632 & rxy $>$ rvalue & Valid \\
\hline 2 & 0.80 & 0.632 & rxy $>$ rvalue & Valid \\
\hline 3 & 0.67 & 0.632 & rxy $>$ r value & Valid \\
\hline 4 & 0.83 & 0.632 & rxy $>$ r value & Valid \\
\hline 5 & 0.74 & 0.632 & rxy $>$ r value & Valid \\
\hline 6 & 0.63 & 0.632 & rxy $>$ r value & Valid \\
\hline 7 & 0.65 & 0.632 & rxy $>$ r value & Valid \\
\hline 8 & 0.89 & 0.632 & rxy $>$ r value & Valid \\
\hline 9 & 0.67 & 0.632 & rxy $>$ r value & Valid \\
\hline 10 & 0.78 & 0.632 & rxy $>$ r value & Valid \\
\hline 11 & 0.70 & 0.632 & rxy $>$ r value & Valid \\
\hline 12 & 0.83 & 0.632 & rxy $>$ r value & Valid \\
\hline 13 & 0.92 & 0.632 & rxy $>$ r value & Valid \\
\hline 14 & 0.75 & 0.632 & rxy $>$ r value & Valid \\
\hline 15 & 0.80 & 0.632 & rxy $>$ r value & Valid \\
\hline
\end{tabular}

4. Crossword Noun (Food)

\begin{tabular}{|c|c|c|c|c|}
\hline $\begin{array}{l}\text { Question } \\
\text { Number }\end{array}$ & $\begin{array}{c}\text { Correlation } \\
\text { Coefficient } \\
\text { (rxy) }\end{array}$ & $\begin{array}{c}\mathbf{r} \\
\text { value } \\
5 \%\end{array}$ & $\begin{array}{c}\text { Comparative } \\
\text { between rxy and } \mathrm{r} \\
\text { value }\end{array}$ & $\begin{array}{c}\begin{array}{c}\text { Category } \\
\text { of }\end{array} \\
\text { Validity }\end{array}$ \\
\hline 1 & 0.70 & 0.632 & rxy $>$ r value & Valid \\
\hline 2 & 0.64 & 0.632 & rxy $>$ r value & Valid \\
\hline 3 & 0.65 & 0.632 & rxy $>$ r value & Valid \\
\hline 4 & 0.64 & 0.632 & rxy $>$ r value & Valid \\
\hline 5 & 0.78 & 0.632 & rxy $>$ r value & Valid \\
\hline 6 & 0.66 & 0.632 & rxy $>$ r value & Valid \\
\hline 7 & 0.63 & 0.632 & rxy $>$ r value & Valid \\
\hline 8 & 0.66 & 0.632 & rxy $>$ r value & Valid \\
\hline 9 & 0.76 & 0.632 & rxy $>$ r value & Valid \\
\hline 10 & 0.67 & 0.632 & rxy $>$ r value & Valid \\
\hline
\end{tabular}




\begin{tabular}{|l|l|l|l|l|}
\hline 11 & 0.67 & 0.632 & rxy $>$ rvalue & Valid \\
\hline 12 & 0.70 & 0.632 & rxy $>$ rvalue & Valid \\
\hline 13 & 0.88 & 0.632 & rxy $>$ rvalue & Valid \\
\hline 14 & 0.68 & 0.632 & rxy $>$ rvalue & Valid \\
\hline 15 & 0.86 & 0.632 & rxy $>$ rvalue & Valid \\
\hline
\end{tabular}

5. Crossword Noun (Animal)

\begin{tabular}{|c|c|c|c|c|}
\hline $\begin{array}{l}\text { Question } \\
\text { Number }\end{array}$ & $\begin{array}{c}\text { Correlation } \\
\text { Coefficient } \\
\quad(\mathrm{rxy}) \\
\end{array}$ & $\begin{array}{c}\mathrm{r} \\
\text { value } \\
5 \%\end{array}$ & $\begin{array}{c}\text { Comparative } \\
\text { between rxy and } \mathbf{r} \\
\text { value }\end{array}$ & $\begin{array}{c}\begin{array}{c}\text { Category } \\
\text { of }\end{array} \\
\text { Validity }\end{array}$ \\
\hline 1 & 0.73 & 0.632 & rxy $>$ rvalue & Valid \\
\hline 2 & 0.65 & 0.632 & rxy $>$ r value & Valid \\
\hline 3 & 0.76 & 0.632 & rxy $>$ rvalue & Valid \\
\hline 4 & 0.63 & 0.632 & rxy $>$ r value & Valid \\
\hline 5 & 0.65 & 0.632 & rxy $>$ r value & Valid \\
\hline 6 & 0.73 & 0.632 & rxy $>$ rvalue & Valid \\
\hline 7 & 0.75 & 0.632 & rxy $>$ r value & Valid \\
\hline 8 & 0.67 & 0.632 & rxy $>$ rvalue & Valid \\
\hline 9 & 0.75 & 0.632 & rxy $>$ rvalue & Valid \\
\hline 10 & 0.88 & 0.632 & rxy $>$ rvalue & Valid \\
\hline 11 & 0.83 & 0.632 & rxy $>$ r value & Valid \\
\hline 12 & 0.93 & 0.632 & rxy $>$ rvalue & Valid \\
\hline 13 & 0.68 & 0.632 & rxy $>$ r value & Valid \\
\hline 14 & 0.90 & 0.632 & rxy $>$ rvalue & Valid \\
\hline 15 & 0.87 & 0.632 & rxy $>$ rvalue & Valid \\
\hline
\end{tabular}

6. Crossword Academic Writing

\begin{tabular}{|c|c|c|c|c|}
\hline $\begin{array}{l}\text { Question } \\
\text { Number }\end{array}$ & $\begin{array}{c}\text { Correlation } \\
\text { Coefficient } \\
\text { (rxy) }\end{array}$ & $\begin{array}{c}r \\
\text { value } \\
5 \%\end{array}$ & $\begin{array}{c}\text { Comparative } \\
\text { between rxy and } r \\
\text { value }\end{array}$ & $\begin{array}{c}\begin{array}{c}\text { Category } \\
\text { of }\end{array} \\
\text { Validity }\end{array}$ \\
\hline 1 & 0.76 & 0.632 & rxy $>$ rvalue & Valid \\
\hline 2 & 0.65 & 0.632 & rxy $>$ r value & Valid \\
\hline 3 & 0.70 & 0.632 & rxy $>$ r value & Valid \\
\hline 4 & 0.70 & 0.632 & rxy $>$ r value & Valid \\
\hline 5 & 0.64 & 0.632 & rxy $>$ r value & Valid \\
\hline 6 & 0.80 & 0.632 & rxy $>$ r value & Valid \\
\hline 7 & 0.78 & 0.632 & rxy $>$ r value & Valid \\
\hline 8 & 0.80 & 0.632 & rxy $>$ r value & Valid \\
\hline 9 & 0.83 & 0.632 & rxy $>$ r value & Valid \\
\hline
\end{tabular}


Sukirman \& Asti Astuti Ningsih,Designing Crossword Puzzle to Improve Students' Vocabulary Mastery ...

\begin{tabular}{|l|l|l|l|l|}
10 & 0.95 & 0.632 & rxy $>$ rvalue & Valid \\
\hline 11 & 0.69 & 0.632 & rxy $>$ r value & Valid \\
\hline 12 & 0.72 & 0.632 & rxy $>$ r value & Valid \\
\hline 13 & 0.87 & 0.632 & rxy $>$ r value & Valid \\
\hline 14 & 0.88 & 0.632 & rxy $>$ rvalue & Valid \\
\hline 15 & 0.69 & 0.632 & rxy $>$ rvalue & Valid \\
\hline
\end{tabular}

Based on the table about crossword puzzle validity above, the researcher had gathered that from the 90 number of questions, divided in six kinds of crossword puzzle and each kind of crossword were consist of 15 number were valid.

2) Realibility

The Result ofRealibility of first model crossword puzzle first model

\begin{tabular}{|c|l|c|}
\hline No. & \multicolumn{1}{|c|}{ Kind of Crossword } & Realibility \\
\hline 1 & Crossword puzzle adjective & 0.79 \\
\hline 2 & Crossword puzzle verb & 0.81 \\
\hline 3 & $\begin{array}{l}\text { Crossword puzzle noun } \\
\text { (abstract) }\end{array}$ & 0.80 \\
\hline 4 & $\begin{array}{l}\text { Crossword puzzle noun } \\
\text { (people) }\end{array}$ & 0.80 \\
\hline 5 & Crossword puzzle noun (place) & 0.87 \\
\hline 6 & $\begin{array}{l}\text { Crossword puzzle noun } \\
\text { (nature) }\end{array}$ & 0.80 \\
\hline
\end{tabular}

Based on the table above, the researcher then compared all the reliability values with the $\mathrm{r}_{\text {table }}$ value of Product Moment table. In the table of Product Moment, for testee amount $(\mathrm{df})=10$ with significant index $5 \%$, the value of $\mathrm{r}_{\text {table }}=0.632$. The comparison shows that all the reliability values of the crossword puzzle were higher than or " $>$ " value

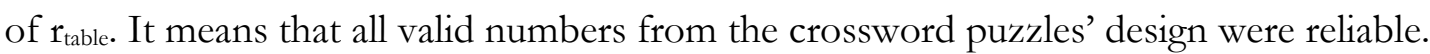

The Result ofRealibility of second model crossword puzzle first model

\begin{tabular}{|c|l|c|}
\hline No. & \multicolumn{1}{|c|}{ Kind of Crossword } & Realibility \\
\hline 1 & Crossword puzzle verb I & 0.78 \\
\hline 2 & Crossword puzzle verb II & 0.80 \\
\hline 3 & Crossword puzzle noun & 0.82 \\
\hline 4 & Crossword puzzle noun (food) & 0.90 \\
\hline 5 & $\begin{array}{l}\text { Crossword puzzle noun } \\
\text { (animal) }\end{array}$ & 0.80 \\
\hline 6 & $\begin{array}{l}\text { Crossword puzzle academic } \\
\text { writing }\end{array}$ & 0.86 \\
\hline
\end{tabular}

Based on the table above, the researcher then compared all the reliability values with the $\mathrm{r}_{\text {table }}$ value of Product Moment table. In the table of Product Moment, for testee amount $(\mathrm{df})=10$ with significant index $5 \%$, the value of $r_{\text {table }}=0.632$. The comparison shows that all the reliability values of the crossword puzzle were higher than or " $>$ " value 
of $\mathrm{r}_{\text {table. }}$ It means that all valid numbers from the crossword puzzles' design were reliable.

\section{Final Product}

The final product was the crossword puzzle design which consists of 12 puzzles and 150 number of questions. In the first model of a crossword puzzle, there were 60 numbers of valid and reliable questions. Then the second model of a crossword puzzle, there were 90 numbers of valid and reliable questions. The crossword puzzle was used to help students learning vocabulary with easier than before and also as a measure of students' improvement in vocabulary mastery. In this design, researcher focused to help students improve their vocabulary mastery of common vocabulary such as adjectives, verb (irregular and regular), noun (abstract, people, place, nature, food, animal, etc), and also academic writing.

\section{E. CONCLUSION}

One of appropriate method for improving students' vocabulary mastery for the third semester's students of the English Education Department at UIN Alauddin Makassar is crossword puzzle. The crossword designs consist of two models. The first model consist of ten kinds of crossword puzzle and each puzzle contain of ten questions. The vocabularies that researcher used are adjective, irregular verb, regular verb, noun (nature), noun (mostly abstract), noun (place), and noun (people). The second model consist of ten kinds of crossword puzzle and each puzzle consist of fifteen questions. The vocabulary thet researcher used are regular verb, irregular verb, verb, noun (common), noun (food), noun (animal), and academic writing.

From the research, the researcher also found the weaknesses from the crossword puzzle. The weaknesses is only when the researcher give many puzzle to students. It make students feel hard to answer all questions because they see too many puzzle. So that to minimize it, the researcher suggest to give the crossword puzzle step by step according to the theme of vocabulary that students learn at the day. For example, today the students learning about verb, so the crossword puzzle that will teacher gives to students only about corossword puzzle verb.

Based on the explanation above, one of the appropriate method to improve students' vacabulary mastery is crossword puzzle. And crossword puzzle that researcher design and develop are ready to be implemented in the learning vocabulary class.

\section{F. SUGGESTION}

For the English teacher, the first thing that we have to know is students' need. It is the main point that the teacher should pay attention. When a teacher tries to look for what students' need than they use an appropriate method based on students' need. It will help students to learn easier than before. 
For teacher, researcher, or reader, which want to develop or designing crossword puzzle, it is advisable to consult with the expert about how to create an appropriate design. Besides, there are still many interesting games that we can design for help students in learning English Language easier than before.

\section{G. APPENDICES}

\section{Adjective}

\section{Directions :}

1. Answer the questions above with an appropriate question.

2. Answer the question in the appropriate column.

3. There will be to model of questions, its down and across.

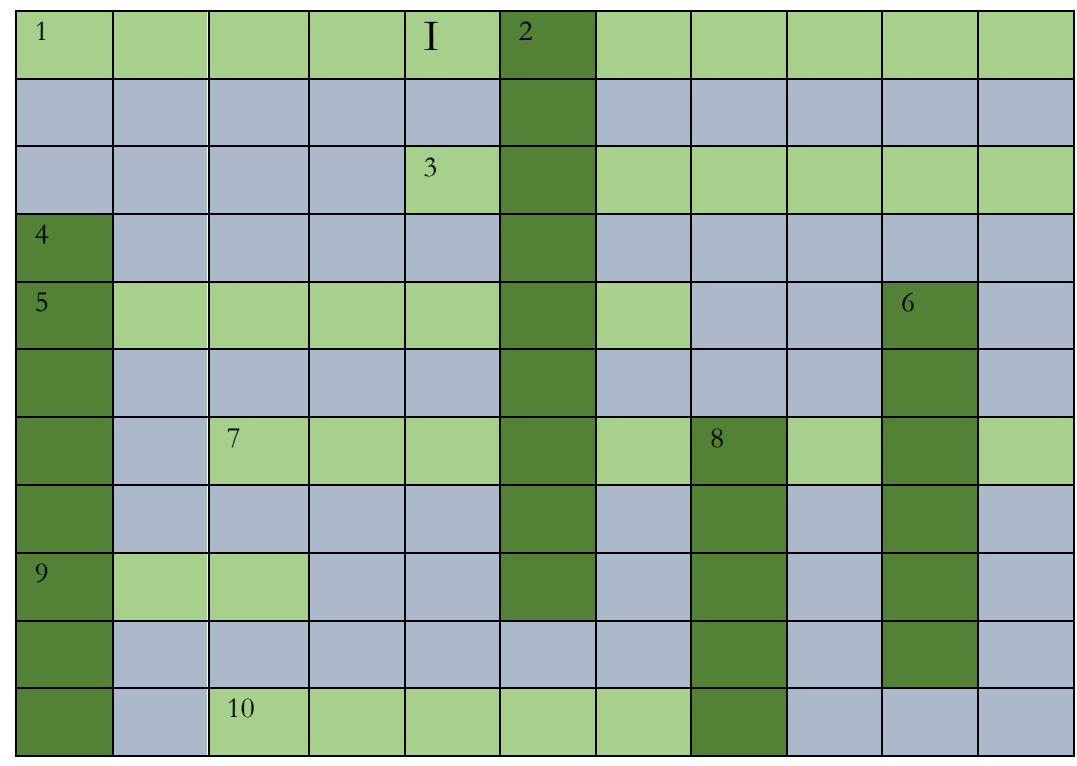

\section{A. Down}

2. Receiving good from uncertain or unexpected sources, lucky.

4. Showing, using or characterized by caution

6. Pleasurable entertained, occupied.

8. Having or causing an itching sensation.

\section{B. Across}

1. Making a splendid appereance or show; of exceptional beauty, size, etc.

3. Full of armed with prickles.

5. Full of mental distress or uneasiness because of fear of danger

7. Full of spirit, dynamic. 
9. Differing in nature from what is ordinary.

10. Wrong especially against moral or penal low.

\section{DIRECTIONS:}

A. Look for a hide vocabulary in the puzzle below

B. Cross the vocabulary that you find or give it color.

\begin{tabular}{|c|c|c|c|c|c|c|c|c|c|c|c|c|c|c|c|c|c|c|c|c|c|c|c|}
\hline Z & $\mathrm{X}$ & $\mathrm{R}$ & B & $\mathrm{Q}$ & W & $\mathrm{E}$ & $\mathrm{R}$ & $\mathrm{T}$ & I & $\mathrm{T}$ & A & S & $\mathrm{R}$ & $\mathrm{T}$ & V & $\mathrm{C}$ & B & $\mathrm{K}$ & I & $\mathrm{L}$ & $\mathrm{O}$ & $\mathrm{P}$ & B \\
\hline $\mathrm{C}$ & $\mathrm{W}$ & $\mathrm{T}$ & $\mathrm{U}$ & I & $\mathrm{U}$ & $\mathrm{Q}$ & $Y$ & $\mathrm{H}$ & B & $\mathrm{E}$ & B & $\mathrm{K}$ & $\mathrm{L}$ & I & $\mathrm{U}$ & $\mathrm{T}$ & S & $\mathrm{P}$ & $\mathrm{L}$ & $\mathrm{N}$ & $\mathrm{P}$ & $\mathrm{R}$ & $\mathrm{P}$ \\
\hline $\mathrm{V}$ & $\mathrm{R}$ & $\mathrm{G}$ & $\mathrm{I}$ & $\mathrm{F}$ & D & $\mathrm{R}$ & $\mathrm{E}$ & $\mathrm{E}$ & $\mathrm{L}$ & A & $\mathrm{L}$ & $\mathrm{E}$ & $A$ & $\mathrm{R}$ & $\mathrm{N}$ & $\mathrm{V}$ & I & $\mathrm{T}$ & $\mathrm{E}$ & $\mathrm{L}$ & $\mathrm{O}$ & $\mathrm{S}$ & $\mathrm{E}$ \\
\hline B & $\mathrm{T}$ & $\mathrm{S}$ & $\mathrm{D}$ & W & $\mathrm{E}$ & $\mathrm{R}$ & $\mathrm{Q}$ & $\mathrm{U}$ & $\mathrm{E}$ & $\mathrm{R}$ & $\mathrm{O}$ & A & S & $\mathrm{T}$ & $\mathrm{E}$ & $\mathrm{R}$ & $S$ & $\mathrm{~T}$ & $\mathrm{E}$ & $\mathrm{A}$ & $A$ & I & $\mathrm{R}$ \\
\hline$G$ & $\mathrm{U}$ & $\mathrm{F}$ & I & $G$ & $\mathrm{U}$ & $\mathrm{E}$ & $\mathrm{R}$ & $\mathrm{E}$ & $\mathrm{D}$ & Y & $\mathrm{H}$ & $\mathrm{O}$ & $\mathrm{L}$ & $\mathrm{L}$ & $\mathrm{D}$ & $\mathrm{E}$ & $\mathrm{A}$ & $\mathrm{R}$ & $\mathrm{D}$ & $\mathrm{L}$ & $\mathrm{O}$ & $\mathrm{A}$ & $\mathrm{N}$ \\
\hline $\mathrm{T}$ & I & $\mathrm{R}$ & $\mathrm{G}$ & $\mathrm{E}$ & $\mathrm{R}$ & $\mathrm{H}$ & I & $\mathrm{T}$ & $\mathrm{O}$ & $\mathrm{E}$ & S & I & $\mathrm{N}$ & S & $\mathrm{E}$ & $\mathrm{C}$ & $\mathrm{T}$ & $\mathrm{C}$ & $\mathrm{U}$ & $\mathrm{L}$ & $\mathrm{L}$ & $\mathrm{S}$ & $\mathrm{N}$ \\
\hline $\mathrm{Y}$ & $\mathrm{P}$ & $\mathrm{E}$ & $\mathrm{N}$ & $G$ & $\mathrm{U}$ & I & $\mathrm{N}$ & I & G & $\mathrm{H}$ & $\mathrm{T}$ & $\mathrm{S}$ & $\mathrm{T}$ & $\mathrm{A}$ & $\mathrm{L}$ & $\mathrm{L}$ & $\mathrm{A}$ & $S$ & $\mathrm{~T}$ & $\mathrm{Y}$ & $\mathrm{H}$ & I & $\mathrm{S}$ \\
\hline $\mathrm{U}$ & I & $\mathrm{E}$ & $\mathrm{N}$ & $G$ & $\mathrm{~L}$ & I & $\mathrm{G}$ & S & $\mathrm{H}$ & $\mathrm{E}$ & $\mathrm{F}$ & $\mathrm{E}$ & $\mathrm{L}$ & $\mathrm{L}$ & $\mathrm{O}$ & $\mathrm{S}$ & $\mathrm{C}$ & $\mathrm{H}$ & $\mathrm{C}$ & $\mathrm{O}$ & $\mathrm{O}$ & $\mathrm{L}$ & I \\
\hline I & $\mathrm{U}$ & Z & $\mathrm{C}$ & I & $\mathrm{N}$ & $\mathrm{T}$ & A & I & $\mathbf{n}$ & $\mathrm{O}$ & A & 1 & $\mathrm{G}$ & $\mathrm{O}$ & $\mathrm{T}$ & $\mathrm{O}$ & W & $\mathrm{N}$ & W & A & $\mathrm{L}$ & L & $\mathrm{E}$ \\
\hline $\mathrm{O}$ & $\mathrm{F}$ & $\mathrm{E}$ & $\mathrm{L}$ & $\mathrm{L}$ & W & $\mathrm{E}$ & A & $\mathrm{R}$ & $\mathrm{O}$ & S & $\mathrm{L}$ & A & $\mathrm{D}$ & I & $\mathrm{E}$ & S & B & $A$ & $\mathrm{D}$ & D & $\mathrm{Y}$ & $\mathrm{N}$ & S \\
\hline $\mathrm{C}$ & $\mathrm{O}$ & $\mathrm{O}$ & $\mathrm{L}$ & D & W & $A$ & $\mathrm{~T}$ & $\mathrm{E}$ & $\mathrm{R}$ & S & $\mathrm{L}$ & $\mathrm{I}$ & $\mathrm{S}$ & $\mathrm{T}$ & $\mathrm{V}$ & $\mathrm{E}$ & $\mathrm{R}$ & $\mathrm{T}$ & $\mathrm{D}$ & $\mathrm{R}$ & $\mathrm{E}$ & $\mathrm{A}$ & $\mathrm{M}$ \\
\hline $\mathrm{P}$ & $\mathrm{A}$ & $\mathrm{V}$ & B & $\mathrm{N}$ & $\mathrm{C}$ & $\mathrm{O}$ & $\mathrm{S}$ & $\mathrm{T}$ & $\mathrm{L}$ & $\mathrm{O}$ & $\mathrm{P}$ & $\mathrm{E}$ & $\mathrm{R}$ & $S$ & $\mathrm{~L}$ & $\mathrm{~A}$ & $\mathrm{Y}$ & $\mathrm{C}$ & $\mathrm{V}$ & $E$ & $\mathrm{R}$ & $\mathrm{T}$ & $\mathrm{U}$ \\
\hline $\mathrm{E}$ & A & $\mathrm{T}$ & $\mathrm{L}$ & $\mathrm{K}$ & $\mathrm{J}$ & $Y$ & $\mathrm{~T}$ & $\mathrm{U}$ & I & $\mathrm{O}$ & $\mathrm{P}$ & $S$ & $\mathrm{E}$ & $\mathrm{A}$ & $\mathrm{R}$ & $\mathrm{T}$ & $\mathrm{C}$ & $\mathrm{F}$ & $\mathrm{K}$ & $\mathrm{P}$ & $\mathrm{P}$ & $\mathrm{O}$ & $\mathrm{L}$ \\
\hline $\mathrm{R}$ & $\mathrm{V}$ & $\mathrm{E}$ & $\mathrm{C}$ & $\mathrm{R}$ & $\mathrm{T}$ & $\mathrm{U}$ & $\mathrm{P}$ & $\mathrm{E}$ & $\mathrm{R}$ & S & $\mathrm{U}$ & B & $\mathrm{N}$ & $\mathrm{T}$ & $\mathrm{H}$ & $\mathrm{R}$ & $\mathrm{O}$ & W & $\mathrm{V}$ & I & $\mathrm{U}$ & $\mathrm{J}$ & I \\
\hline $\mathrm{T}$ & $\mathrm{E}$ & $A$ & $S$ & $\mathrm{H}$ & $\mathrm{U}$ & $\mathrm{S}$ & $\mathrm{H}$ & $\mathrm{U}$ & 1 & $\mathrm{C}$ & 3 & 1 & $\mathrm{E}$ & $\mathrm{R}$ & $\mathrm{T}$ & I & $\mathrm{A}$ & $\mathrm{L}$ & $S$ & $\mathrm{~V}$ & B & $\mathrm{T}$ & $\mathrm{E}$ \\
\hline
\end{tabular}

\section{REFERENCES}

Arikunto, Suharsimi. Prosedur Penilitian. Jakarta: Rineka Cipta, 2013.

Befridge. Games For Language Learning. Cambridge: Cambridge University. 1984.

Borg, W.R \& Call M.D. Educational Research An Introduction. New York: Longman, 1983.

Bressan, D. Crossword Puzzle in Modern Language Teaching. Audio-Visual Language Journal. 1970.

Gay, L.R. Educational Research Competence for Analysis and Application. Boston: Newbury: 
Sukirman \& Asti Astuti Ningsih,Designing Crossword Puzzle to Improve Students' Vocabulary Mastery ...

Mersil Company. 1982.

Groundlund, N.Es. Measurement and Evaluation testing ( $5^{\text {th }}$ Ed). New York: Macmillan Publishing, 1990.

Harmer, J. The Practice of English Language Teaching. USA: Longman. 2008.

Hatch and Farhady, H. Research Design and Statistic for Applied Linguistic. Rowley Massachussets: Newbury House Publisher Inc.

Hornby. Advanced Learner Dictinary of English. Oxford: Oxford University Press. 1987.

Latief, M.A. Research Methods on Language Learning An Introduction. Malang: UM Press, 2013.

Lattore, G. The Contribution and the Use of EFL Crossword Puzzle. ELT Journal Volume XXX. 1975.

Mohana, MS. English Language: Growth of Vocabulary. Saudi Arabia: ARC Journal, 2013

Marshan, S. (2015). Developing English Morphology Materials For Undergraduate Students At Alauddin State Islamic University Of Makassar. ETERNAL (English, Teaching, Learning, and Research Journal), 1(1), 1-14.

Mubar, M. K. N. A. (2015). Developing English Learning Materials For Young Learners Based On Needs Analysis At Mtsn Model Makassar. ETERNAL (English, Teaching, Learning, and Research Journal), 1(2), 313-330.

Mulyasa, E. (2013). Pengembangan dan Implementasi Kurikulum 2013: Perubahan dan Pengembangan Kurikulum 2013 Merupakan Persoalan Penting dan Genting. hlm. 65.

Nation \& Macalister.(2009) . Language Design Curriculum. Taylor \& Francis e-Library.

Nurpahmi, S. (2013). An Introduction to English for Specific Purposes. Makassar: Alauddin University Press.

Nurpahmi, S. (2014). English for Specific Purposes: An Integrated Approach. Makassar: Alauddin University Press.

Nurpahmi, S. (2017). ESP Course Design: An Integrated Approach. Lentera Pendidikan: Jurnal Ilmu Tarbiyah Dan Keguruan, 19(2), 172-181.

Njoro, C.Martin, etc. The Use of Crossword Puzzle as a Vocabulary Learning Strategy: A Case of English as a Second Language in Kenyan Secondary School. Kenya: International Journal of Current Research, 2013.

Putra, Nusa. Researche Development. Jakarta: RajaGrafindo Persada, 2012

Sri, Wahyuni. Designing English Tests for Psychomotor Domain dealing with the 2013 Curriculum at the First Year Students of SMAN 1 Makassa, Skripsi at UIN Alauddin University. Makassar. 2015. 
Volume 02, Number 01, June 2016 